\title{
Fuzzy Visa: um modelo de lógica fuzzy para a avaliação de risco da Vigilância Sanitária para inspeção de resíduos de serviços de saúde
}

I ${ }^{1}$ Natércia Fonseca de Carvalho da Silva, ${ }^{2}$ Cid Manso de Mello Vianna,

${ }^{3}$ Fabiano Saldanha Gomes de Oliveira, ${ }^{4}$ Gabriela Bittencourt Gonzalez Mosegui,

${ }^{5}$ Marcus Paulo da Silva Rodrigues I

Resumo: A inspeção sanitária de Gerenciamento de Resíduos de Serviços de Saúde (GRSS) carece de metodologia de trabalho padronizada que proporcione bases seguras de avaliação de risco potencial (RP). O objetivo deste trabalho foi propor um instrumento baseado em lógica fuzzy capaz de padronizar a inspeção e gerar indicadores de controle sanitário. O modelo foi desenvolvido com 18 subsistemas relacionados ao GRSS, que resultaram da aglutinação de elementos identificados na inspeção, como aqueles que interferem na avaliação e permeiam todas as etapas do GRSS. A validação do modelo foi realizada em dez unidades de saúde no Rio de Janeiro, de maio a novembro de 2009. Os resultados obtidos no sistema seguiram os diagnósticos realizados pelos especialistas, mostrando a possibilidade de sistematização e racionalização das avaliações de RP. Esse sistema de apoio à decisão torna mais eficiente a gestão e o planejamento de açôes na avaliação do RP.

> Palavras-chave: Resíduos de serviços de saúde; lógica fuzzy; vigilância sanitária; inspeção sanitária; técnicas de apoio à decisão.
1 Instituto Estadual do

Ambiente do Rio de Janeiro. Rio de Janeiro-RJ, Brasil

(nfonseca2013@gmail.com)

${ }^{2}$ Instituto de Medicina Social, Universidade do Estado do Rio de Janeiro. Rio de Janeiro-RJ,

Brasil (cmmv@ims.uerj.br).

${ }^{3}$ Instituto de Medicina Social, Universidade do Estado do Rio de Janeiro. Rio de Janeiro-RJ, Brasil (fgomes@Incc.br).

${ }^{4}$ Instituto de Saúde Coletiva, Universidade Federal Fluminense. Niterói-RJ, Brasil (gabrielamosegui@uol.com.br).

${ }^{5}$ Instituto de Medicina Social, Universidade do Estado do Rio de Janeiro. Rio de Janeiro-RJ, Brasil(mps_rodrigues@yahoo. com.br).

Recebido em: 27/10/2015 Aprovado em: 02/08/2016 


\section{Introdução}

Embora o avanço tecnológico tenha possibilitado surpreendentes conquistas no campo das ciências, também contribuiu para o aumento da diversidade de produtos e materiais de difícil degradação e maior toxidade, que ao fim da sua cadeia de utilização, invariavelmente, se tornam resíduos. A gestão inapropriada desses resíduos traz claras implicaçōes à saúde humana e ao meio ambiente, tais como contaminação do solo, das águas superficiais e subterrâneas e do ar. O objetivo do gerenciamento é alcançar a excelência em todas as etapas e processos envolvidos, como na segregação e identificação dos resíduos, assim como no transporte adequado e destinação final, entre outros. Identifica-se claramente a necessidade de atenção com cada ação específica, traduzindo-se na melhoria da avaliação do nível de adequação dos EAS, o que reduz o risco potencial.

A Vigilância Sanitária do Estado do Rio de Janeiro, em suas ações de fiscalização e orientação, tem-se empenhado em avaliar não apenas o gerenciamento interno dos resíduos (intramuros), mas também garantir que os estabelecimentos acompanhem e interajam nas etapas que são executadas na sequência extramuro (de coleta e transporte, tratamento e destino final). Isto significa envolver, em um mesmo processo de gerenciamento de RSS, estabelecimentos que não são os geradores dos resíduos, mas que participam do processo de gerenciamento, como transportadores e receptores, estimulando o desenvolvimento de uma cultura de proteção ao meio ambiente.

A fim de salvaguardar a saúde da população e a proteção ao meio ambiente, o gerenciamento dos resíduos, como os gerados nos serviços de saúde, vem sendo objeto de preocupação e regulação da vigilância sanitária. Ainda que constituam uma pequena parcela da massa total de resíduos sólidos urbanos, suas características físicas, químicas ou infecto-contagiosas podem agregar um grande número de fatores de risco à saúde pública e ao meio ambiente. A fiscalização sanitária, através dos preceitos das legislaçôes específicas, é instrumento fundamental das ações de proteção da saúde e ao ambiente.

A vigilância sobre os resíduos de serviços de saúde (RSS) se faz cada vez mais necessária porque os estabelecimentos assistenciais de saúde (EAS) têm agregado novas tecnologias em seus processos de trabalho e ampliado sua complexidade. 
Como consequência, a quantidade e as características dos resíduos gerados têm aumentado, ampliando também o risco, sendo necessário aprimorar as técnicas de gerenciamento interno e externo, de coleta, de transporte, de tratamento e de destino final dos resíduos.

As práticas de vigilância sanitária se estabelecem com base na noção de risco, perigo virtual ou ameaça de agravo. Esta noção se relaciona com determinados elementos que o homem aprendeu a identificar na experiência cotidiana (COSTA, 1998;2004). Apreender as condiçõos que assumem os riscos e incorporar de forma científica as avaliações realizadas, com base na experiência profissional e nas incertezas epistêmicas dos riscos, nos objetos sob seu controle, conforma um desafio sempre presente nas práticas diárias de vigilância sanitária. Situações reais exigem avaliação e tomada de decisão (COSTA, 2004), com base no risco ou perigo, muitas vezes apresentados em condições "difusas". Na prática da vigilância sanitária, esta noção de "risco potencial" exprime a possibilidade de ocorrência de um evento danoso à saúde, independentemente da probabilidade de ocorrência ou da severidade do dano (LEITE, 2007).

Não existe nenhum instrumento estabelecido no Brasil para as inspeções sanitárias de RSS. No Estado do Rio de Janeiro, não se utilizanenhum instrumento formal, como roteiros e questionários, e tais inspeçôes consistem na observação sistemática dos setores já existentes no EAS, verificando o processo de trabalho empregado, a geração dos resíduos e como se desenvolvem as etapas do seu manejo (segregação, acondicionamento, identificação, armazenamento interno e externo, tratamento e transporte interno e externo e disposição final). Adicionalmente, a inspeção visual também é utilizada para avaliar a adequação e a manutenção da estrutura física e dos equipamentos, sobretudo os empregados no manejo dos RSS ou que se relacionam ao seu gerenciamento, assim como os instrumentos de proteção à saúde dos trabalhadores e os documentos obrigatórios para autorização de funcionamento do estabelecimento, como as licenças ambientais e sanitárias, contratos de serviços terceirizados que possam influenciar no gerenciamento dos RSS (GRSS), programas de treinamento e outros.

A proposta deste trabalho é elaborar um instrumento sob a forma de sistema de informação, para apoiar a tomada de decisão resultante da avaliação do RSS da vigilância sanitária. $\mathrm{O}$ modelo em que se baseia o instrumento é a lógica 
fuzzy, ${ }^{1}$ cuja aplicação em sistemas de apoio à decisão na área da saúde é recente. $\mathrm{O}$ trabalho de Amorim et al. (2015) apresenta um sistema de apoio à decisão baseado na tecnologia de um sistema lógico fuzzy, que processa observações das variáveis em linguagem natural ou linguísticas. Os seres humanos avaliam com maior precisão quando usam linguagem natural do que quando usam escalas de valores numéricos ou categóricos. A avaliação de risco será mais precisa se as observações coletadas forem valores linguísticos.

Os sistemas especialistas usando tecnologias do tipo sistemas lógicos fuzzy integram o ramo da computação conhecido como inteligência artificial/ inteligência computacional. Foram inicialmente usados na área de saúde como geradores de diagnósticos para enfermidade (JANG; SUN; MIZUNAMI, 1997), os bancos de dados eram pequenos e continham basicamente conjuntos de sintomas. Esse tipo de sistema inteligente processava os dados na tentativa de identificar a enfermidade.

$\mathrm{O}$ crescimento do poder computacional estendeu sistemas especialistas para sistemas do tipo apoio à tomada de decisão, que utilizam como insumo quantidades imensas de dados (LIMA; CARPINETTI, 2015). Dentre as tecnologias usadas nestes sistemas está o sistema de lógica fuzzy, que produz inferências sobre os valores das variáveis usando como entrada observações linguísticas. Estas representam a opinião do especialista sobre o fenômeno avaliado.

Experimentos mostram que os seres humanos ranqueiam com maior precisão quando pensam em termos de linguagem natural (AMORIM, 2015), o que melhora a eficácia da avaliação do fenômeno. $\mathrm{O}$ avaliador responde não um valor numérico ou categórico, mas um termo linguístico representando o conjunto mais adequado ao qual pertence a observação coletada. Por exemplo: um avaliador observa 30 vezes com um termômetro a temperatura da água em uma bacia e depois avalia a temperatura colocando a mão dentro da bacia. No primeiro caso, ele registra 30 valores numéricos precisos resultantes do aparelho de medida. No segundo, ele responde se a água está fria, morna ou quente, por exemplo. No primeiro caso, temos 30 valores cuja variabilidade é desconhecida. No segundo, informamos um valor linguístico que melhor representa nossa avaliação da temperatura da água. Para um humano, é muito mais fácil e preciso inferir se a água está fria, morna ou quente, do que inferir o valor da sua temperatura. 
A tecnologia conhecida como sistema lógico fuzzy foi desenvolvida por Zadeh (ZADEH, 1965) para inferir informação a partir de nossa impressão do fenômeno. Cada observação de uma variável fuzzy indica o conjunto que melhor representa a variável. A chamada função de pertinência da variável fuzzy indica o grau de crença ou o quanto a observação pertence ao conjunto fuzzy escolhido. As regras lógicas fuzzy constituem um sistema de equações lógicas cujo resultado é chamado de inferência fuzzy. Esse processo de inferência gera o conjunto fuzzy da variável de saída. Finalmente, o procedimento chamado de defuzzyficação calcula, a partir do conjunto gerado, um número (crisp), como resultado da inferência do sistema lógico fuzzy (SLF), que pode ser usado para a tomada de decisão.

Em linhas gerais, o processamento do sistema lógico fuzzy, segue os passos (JANG; SUN; MIZUNAMI, 1997): receber os valores linguísticos observados; processar a inferência fuzzy; defuzzyficar o conjunto fuzzy de saída em um número. O conjunto de observações imprecisas das entradas é convertido em uma tomada de decisão na saída - valor defuzzyficado. As funções de pertinência associadas às variáveis são construídas subjetivamente, a partir do conhecimento dos especialistas sobre o fenômeno (JANG; SUN; MIZUNAMI, 1997).

O sistema FUZZY VISA agrega 18 subsistemas de inferência, sendo cada qual um modelo lógico fuzzy de Mamdani com duas entradas ou antecedentes e um de saída ou consequente (JANG; SUN; MIZUNAMI, 1997). O SLF de Mamdani agrega as regras através do operador lógico OU, modelado pelo operador máximo e, em cada regra, o operador lógico, sendo modelado pelo operador mínimo. O método de "defuzzificação" escolhido foi Centro-deGravidade ou Centro-da-Área (JANG; SUN; MIZUNAMI, 1997).

Este instrumento, o Fuzzy Visa, permite a organização e o processamento de dados que possam ser utilizados para regulação, monitoramento e planejamento da vigilância sanitária. O Fuzzy Visa deverá também ser capaz de fornecer a instituição uma classificação do risco potencial dos estabelecimentos inspecionados, em função das não conformidades observadas. Ele auxilia o acompanhamento do risco potencial do gerenciamento dos resíduos em cada etapa de manejo, além da comparação entre os estabelecimentos em função do risco apresentado. 
O Fuzzy Visa aplicado à inspeção sanitária de RSS busca classificar a condição satisfatória do seu gerenciamento e risco potencial (RP). Ele organiza e processa as observações realizadas em estabelecimentos assistenciais de saúde (EAS) do Estado do Rio de Janeiro.

As decisões da Vigilância Sanitária têm como base o atendimento ao Regulamento Técnico de Gerenciamento de Resíduos de Serviços de Saúde da Agência Nacional de Vigilância Sanitária (ANVISA) a Resolução RDC/ANVISA no 306/04 (BRASIL, 2004). As regras lógicas fuzzy foram construídas através da identificação dos elementos relevantes, comumente observados na inspeção sanitária de RSS. A partir dessas observações, foram adotados pontos de controle nas etapas de manejo dos RSS, chamados de "indicadores de risco".

As observações coletadas originaram-se de dez relatórios de inspeções sobre gerenciamento de RSS, elaborados pela Vigilância Sanitária do Estado do Rio de Janeiro de maio a novembro de 2009. Os dez estabelecimentos assistenciais de saúde foram escolhidos ao acaso. A calibração e validação das funçōes de pertinência das variáveis foram baseadas na experiência dos avaliadores. $\mathrm{O}$ sistema foi desenvolvido com uso do software MatLab/Simulink, utilizando o ToolBox Lógica Fuzzy (JANG; SUN; MIZUNAMI, 1997).

No caso do modelo proposto, as informações foram organizadas conforme a sistematização do olhar especialista da equipe de inspeção de resíduos da Vigilância Sanitária do Estado do Rio de Janeiro. O desenvolvimento do sistema lógico fuzzy (SLF) parte da seleção de indicadores de controle (IC), que são pontos-chave a serem observados. Estes influenciam no sucesso do gerenciamento dos resíduos e, por consequência, no controle do risco potencial (RP) relativo ao gerenciamento dos RSS (GRSS).

As variáveis de entrada e os ICs foram organizados nos seguintes subconjuntos fuzzy: inadequado, pouco adequado, sofrível, adequado e muito adequado. A figura 1 mostra a forma desses conjuntos na função de pertinência dessas variáveis. Para evitar a transição abrupta entre os conceitos e permitir melhor precisão no julgamento, escolheram-se funções de transição suave. Os 18 subsistemas relacionados ao GRSS são os indicadores de controle (ICs) obtidos a partir 
da aglutinação de elementos identificados, cuja presença resulta no sucesso do trabalho sob avaliação. Esses elementos permeiam todas as etapas do processo de gerenciamento dos RSS e expressam as principais não conformidades encontradas nas inspeções de RSS. Estas não conformidades foram avaliadas conforme sua adequação ou grau de pertinência ao indicador: quanto maior a pertinência, maior a adequação do IC aos regulamentos técnicos. Por recomendação dos especialistas, usou-se como suporte para a função de pertinência uma escala de adequação de números reais de 0 a 100. O grau de adequação foi ainda classificado em três faixas para fins de monitoramento, que dividem o eixo horizontal em: satisfatório (acima de $90 \%$ ), satisfatório com restriçōes (entre 50\% e 90\%) e insatisfatório (abaixo de 50\%).

Figura 1. Exemplo de função de pertinência para a variável de saída NAESTRUT

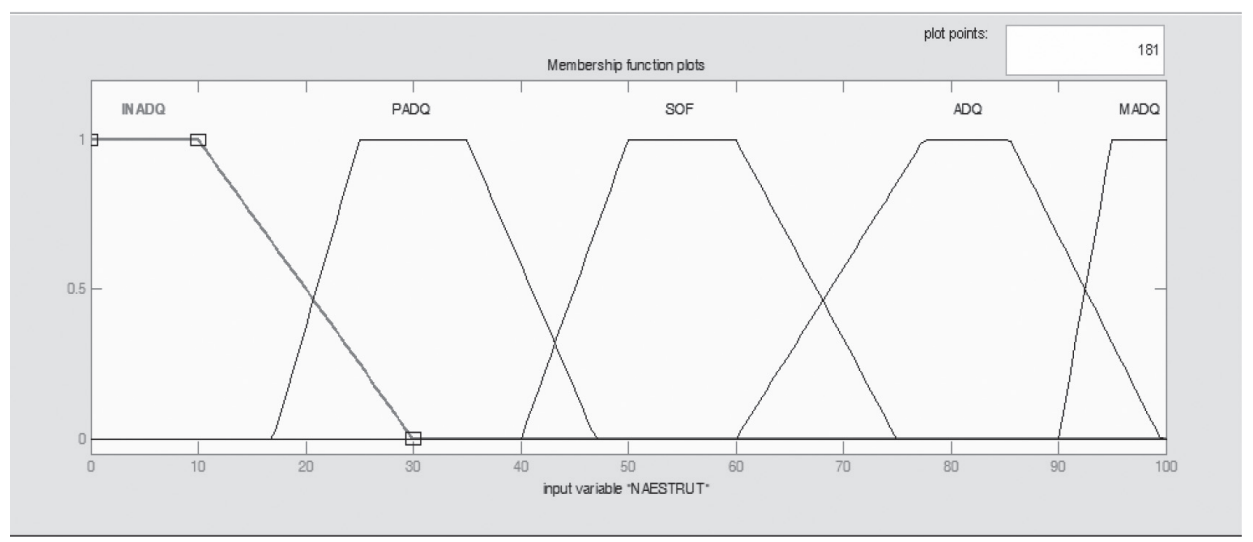

Fonte: elaboração própria.

Com base nas evidências que o agente da Visa dispõe, as operações de inferência fuzzy geram o grau ou nível de adequação do IC. Este grau exprime com maior precisão possível a estrutura de pensamento da equipe de inspeção da Visa (SILVA, 2010; JANG; SUN; MIZUNAMI, 1997). E uma vez estruturada a concepção básica do modelo de avaliação (Fuzzy Visa), a função de pertinência de cada IC foi composta pelos seguintes conjuntos fuzzy: muito adequada (MADQ), adequada (ADQ), sofrível (SOF), pouco adequada (PADQ) e inadequada (INAD). 
A figura 2 apresenta a arquitetura do Fuzzy Visa. O modelo de inferência de cada IC é composto por dois antecedentes e um consequente de Mamdani (SILVA, 2010; JANG; SUN; MIZUNAMI, 1997). O Fuzzy Visa é um SLF com 12 entradas (níveis de adequação) e 18 saídas (valores resultantes da desfuzzyficação do conjunto fuzzy de cada IC). O módulo de inferência fuzzy possui 18 subsistemas lógicos, resultando em um total de 450 regras de inferência fuzzy do tipo se-então (SILVA 2010). O módulo de defuzzyficação converte o conjunto fuzzy de cada IC em seu respectivo valor numérico. Os valores encontrados no vetor de saídas estabelecem, para cada EAS, o resultado da inspeção (tomada de decisão - de insatisfatória a satisfatória).

Figura 2. Arquitetura do modelo Fuzzy Visa

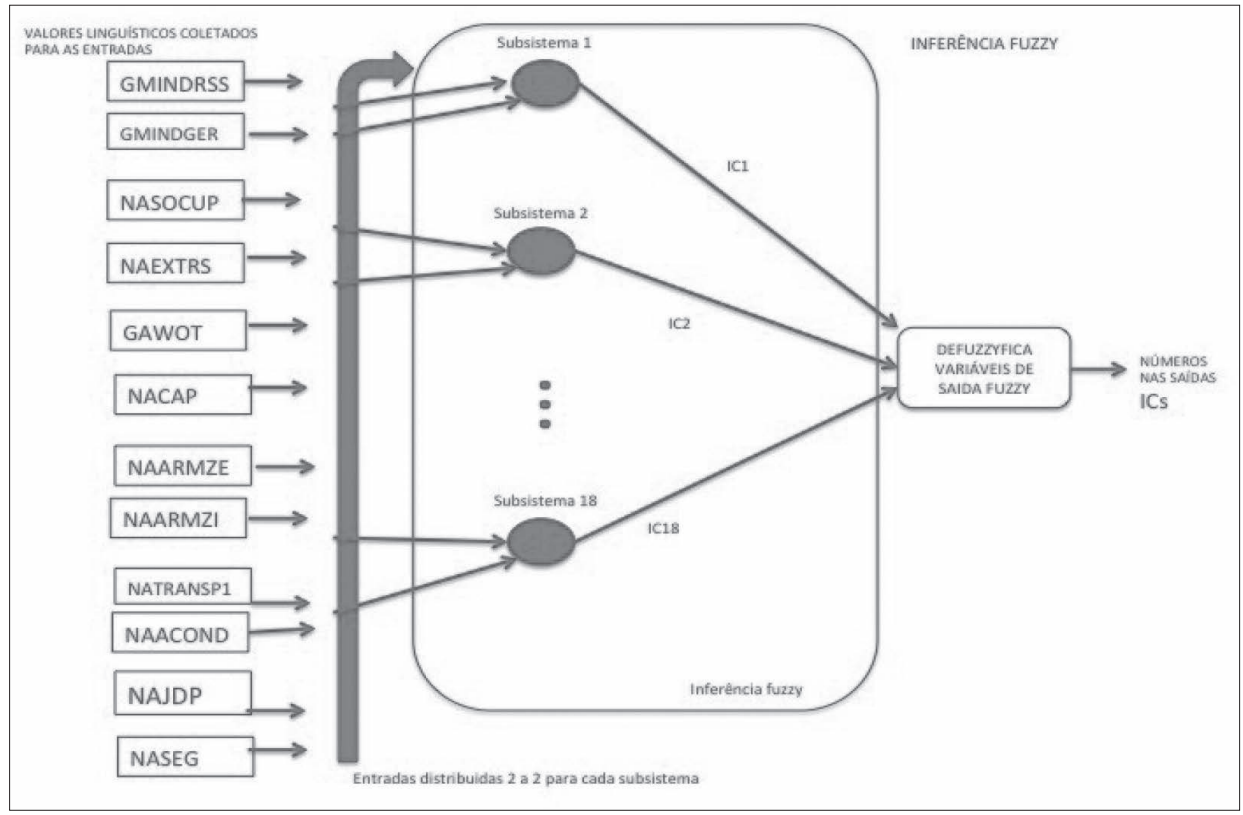

Fonte: elaboração própria.

A figura 3 é uma representação esquemática completa do Fuzzy Visa, que codifica a estrutura de um problema de decisão, por representar explicitamente todas as sequências de possibilidades de observaçooses (entradas) e decisōes (saídas) no modelo. 
As variáveis de entrada foram denominadas de: GMINDGER, GMINDRSS, NASOCUP, NAEXTRS, GAWOT, NACAP, NAARMZI, NAARMZE, NATRANSP1, NAACOND, NASEG e NAJDP. Seus valores foram coletados das respostas dos questionários. Elas representam os níveis de: risco de adequação segregação; acondicionamento; identificação; armazenamento interno e externo; tratamento e transporte interno e externo e disposição final. Os indicadores de controle ICs são: 1-IC1 ou NAGRSS é o nível de adequação do manejo dos RSS e expressa o resultado final da avaliação sobre gerenciamento dos resíduos; 2-IC2 ou NAMRSS representa o nível de adequação do manejo propriamente dito dos resíduos; 3-IC3 ou GHTRAB é o grau de habilitação dos trabalhadores ao trabalho com RSS; 4-IC21 ou NAEMINT representa o nível de adequação das etapas de manejo interno dos RSS; 5-IC22 ou NAEMEXT, o nível de adequação das etapas de manejo externo dos RSS; 6-IC31 ou NAEMPRINC, o nível de adequação das etapas de manejo principal, que compreende as etapas de segregação, identificação, acondicionamento e transporte interno; 7-IC32 ou NAEMSEC é o nível de adequação das etapas de manejo secundário dos RSS, que compreende o armazenamento e o tratamento; 8- IC211B ou NAEM1 é o nível de adequação das primeiras etapas de manejo interno dos resíduos, e 9-IC211A ou NAEM2, as etapas posteriores; 10-IC211 ou NAARMZ, o nível de adequação do armazenamento de uma forma geral; 11-IC212 ou NASATRAB representa o nível de adequação da estrutura que envolve a saúde do trabalhador e do processo e procedimentos empregados na execução do trabalho de uma forma geral; 12-IC221 ou NAPROCTRAB; 13-IC222 ou NAIE é o nível de adequação estrutural geral; 14-IC312 ou NAESTRUT é o nível de adequação da infraestrutura relacionado à estrutura do estabelecimento e dos equipamentos; 15-IC311 ou NAEFIS é o nível de adequação da estrutura física do estabelecimento; 16-ICB1 ou NADOC é o nível de adequação documental; 17-ICA2 ou NAMONITIND é o nível de adequação dos indicadores de monitoramento; 18-ICA1 ou GAEMRSS representa o nível de adequação de desenvolvimento das etapas de manejo dos resíduos, inclusive as etapas extramuro (SILVA, 2010). 


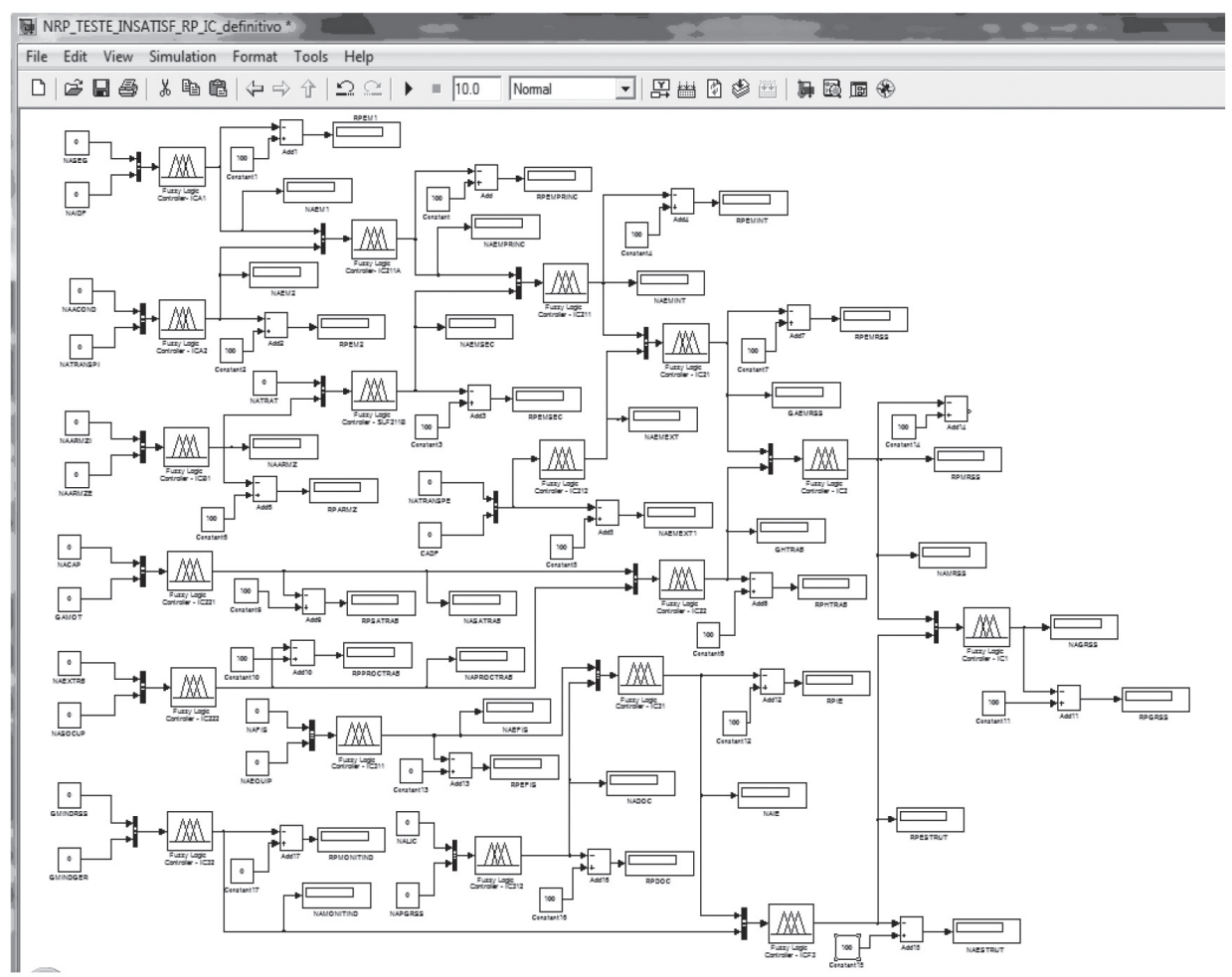

Fonte: elaboração própria.

\section{Validação do modelo}

O modelo desenvolvido foi aplicado na avaliação de risco em dez unidades de saúde do Estado do Rio de Janeiro. As tomadas de decisão suportada pelas respostas do Fuzzy Visa foram comparadas com aquelas expressas nos relatórios de inspeção. Os resultados obtidos pelo modelo demonstram sua coerência e consistência (SILVA, 2010).

A tabela 1 apresenta os resultados das avaliações de risco constantes nos relatórios de inspeção dos EAS 1 a 10, e o nível de adequação do gerenciamento dos RSS obtido através do modelo de avaliação. Os resultados defuzzyficados, ou seja, expressos sob forma numérica, são coerentes e demonstram a consistência do modelo.

Os relatórios de inspeção não apresentam nenhum valor numérico, mas a equipe de inspeção condiciona sua conclusão dentro da percepção de uma escala 
de satisfação. Nessa escala, "0" seria insatisfatório e "100" satisfatório, resultante

de um processo de avaliação amparado nos preceitos legais, principalmente aos contidos na RDC/ANVISA no 306/04, regulamento técnico para o gerenciamento de RSS.

Tabela 1. Resultado encontrado nos relatórios de inspeção e o resultado do modelo

\begin{tabular}{|l|c|c|c|c|}
\hline \multirow{2}{*}{$\begin{array}{c}\text { ESTABELECIMENTO } \\
\text { ASSISTENCIALDE } \\
\text { SAÚDE (EAS) }\end{array}$} & RESULTADO & $\begin{array}{c}\text { VALOR DE } \\
\text { REFERÊNCIA }\end{array}$ & RESULTADO & $\begin{array}{c}\text { VALOR DO } \\
\text { NAGRSS }\end{array}$ \\
\cline { 2 - 5 } EAS 1 & INSATISFATÓRIO & $<50$ & INSATISFATÓRIO & 46,82 \\
\hline EAS2 & INSATISFATÓRIO & $<50$ & INSATISFATÓRIO & 49,51 \\
\hline EAS 3 & INSATISFATÓRIO & $<50$ & INSATISFATÓRIO & 30,7 \\
\hline EAS 4 & INSATISFATÓRIO & $<50$ & INSATISFATÓRIO & 30,07 \\
\hline EAS 5 & INSATISFATÓRIO & $<50$ & INSATISFATÓRIO & 9,019 \\
\hline EAS 6 & SATISFATÓRIO C/ RESTRIIÇÃO & $250<95$ & SATISFATÓRIO C/ RESTRIÇÃO & 74,07 \\
\hline EAS 7 & INSATISFATÓRIO & $<50$ & INSATISFATÓRIO & 30,07 \\
\hline EAS 8 & INSATISFATÓRIO & $<50$ & INSATISFATÓRIO & 30,07 \\
\hline EAS 9 & SATISFATÓRIO C/ RESTRIÇÃO & $250<95$ & SATISFATÓRIO C/RESTRIÇÃO & 79,7 \\
\hline EAS 10 & SATISFATÓRIO C/ RESTRIÇÃO & $\geq 50<95$ & SATISFATÓRIO C/ RESTRIÇÃO & 79,7 \\
\hline
\end{tabular}

Fonte: elaboração própria.

Na inspeção pode ser atribuído um resultado considerado desde insatisfatório a satisfatório. Insatisfatórias são aquelas situações em que a percepção de satisfação, ou seja, de atendimento aos preceitos legais, não atinge o equivalente à metade do exigido. Satisfatório é quando se percebe que o cumprimento das condicionantes legais forma um cenário de baixíssimo risco, com atendimento de todas ou quase todas as condicionantes, com uma percepção equivalente a 95 (na mesma escala de 0 a 100 citada anteriormente). As situações intermediárias são aquelas onde o risco não é alto, sendo possível discriminar uma lista de restrições de sanáveis a curto prazo. Neste caso, considera-se satisfatório com restrição, sendo facilmente identificado, em uma reinspeção, o cumprimento das exigências formuladas.

O cenário considerado ideal baseia-se na adequação do gerenciamento dos RSS, conforme a RDC/ANVISA n³06/04 (BRASIL, 2004). Os itens comumente observados nas inspeções são: documentos, como os de licenciamento ambiental e sanitário; os manifestos de resíduos (documento de controle da cadeia de produção de resíduos, da geração ao destino final); os contratos de serviços terceirizados que se relacionam ou possam interferir no gerenciamento de RSS (GRSS); a 
estrutura física das edificações e os equipamentos utilizados no GRSS; o PGRSS e seu responsável técnico; os cuidados com a saúde do trabalhador: vacinação obrigatória, reconhecimento dos riscos a que está exposto; capacitação, interdição de unidade; e o monitoramento da geração de resíduos; segregação; identificação; acondicionamento, armazenamento, tratamento, coleta e disposição final de resíduos; fatores relevantes na relação saudável entre o homem e o meio ambiente. A avaliação na inspeção colide com a percepção da existência e operação conjunta de todos os fatores citados acima, de forma a produzir um resultado do grau de adequabilidade, que se traduz no risco potencial imputado ao EAS sob avaliação.

O resultado da avaliação do grau de adequabilidade do GRSS está diretamente relacionado ao risco potencial; embora este possa ser igual para EAS diferentes, dificilmente esses estabelecimentos terão as mesmas particularidades operacionais e, portanto, os mesmos condicionantes ao RP. Isto significa que, observados os mesmos fatores, cada inspeção é única e produzirá resultado com base na percepção de diferentes fatores.

O modelo proposto captura o raciocínio utilizado na inspeção e o transforma em resultado objetivo, através de valores numéricos, que permitem mensurar o grau de adequabilidade e o risco potencial. Em seguida, apresenta-se tabela comparativa da avaliação dos resultados do Fuzzy Visa. Com os resultados expressos na tabela 1, é possível identificar uma das vantagens do modelo em relação à forma atual de avaliação, que seria o discernimento da posição que o EAS ocupa na escala numérica de 0 a 100 já mencionada. Hoje é apenas possível visualizar a permanência do EAS em uma faixa bastante ampla de classificação, como satisfatório, satisfatório com restrição e insatisfatório. Nesse contexto, preliminarmente, é possível perceber que, na situação atual em forma de relatório, o resultado identifica como iguais sete estabelecimentos (insatisfatório) e também três outros (satisfatório com restrição).

Esse resultado, por sua vez, não reflete toda a realidade. A utilização apenas da variável linguística, mesmo com valores referenciais, não permite monitoramento e não traduz os diferentes graus de risco potencial de cada EAS. Extrai-se daí, também, a dificuldade futura de quantificar a melhoria ou piora no GRSS. Pela classificação atual, o EAS 2 e o EAS 5, por exemplo, se encontram na mesma faixa insatisfatória, mas observando a tabela 1 é possível perceber que ocupam posições muito distintas. O EAS 2 recebeu a nota 49,51 no modelo 
proposto, muito próxima à satisfatória com restrição $(\geq 50)$; e o EAS 5, a nota 9,019. Isto comprova que o EAS 5 está em uma condição bem mais incipiente do gerenciamento dos RSS e, consequentemente, com maior RP, ${ }^{2}$ demandando atenção maior e ações urgentes a serem aplicadas na melhoria do GRSS. Embora insatisfatório, para o EAS 2 constata-se claramente que sua pontuação está próxima do limite de mudança de classificação, requerendo atenção, mas com um menor esforço, com bem menos ações saneadoras. O EAS 2 poderá atingir um risco potencial aceitável com poucas ações, permitindo a partir daí na busca da melhoria para o cenário ideal.

As tabelas 2 e 3 apresentam uma síntese dos resultados do RP e do nível, grau ou condição de adequação de cada indicador de controle. Ao IC 211 foi aplicada a nota 50 em todos os estabelecimentos, em função da mensuração inconsistente ou inexistente nos relatórios. O valor 50 , na escala numérica, é o que permite maior neutralização do IC com relação à avaliação dos demais - desta forma, reduz-se a possibilidade de interferência no resultado final.

O resultado das inspeçôes de RSS se resume atualmente ao enquadramento em três faixas (satisfatória, satisfatória com restrição e insatisfatória) e a uma lista enumerada de exigências a serem cumpridas pelo estabelecimento, em prazos predeterminados. A aplicação do modelo incorpora às inspeções outros elementos que permitem acompanhar a evolução minuciosa dos EAS, porque trabalha a inspeção em pontos de controle que são expressos em valores numéricos (IC). A tabela 2 demonstra que é possível extrair e monitorar o risco potencial (RP) em cada indicador de controle. Isto mostra claramente qualquer avanço ou retrocesso no GRSS. Além da importância do monitoramento, os indicadores de controle permitem que o estabelecimento conheça onde estão suas principais falhas. Isto permite ao gestor estabelecer as prioridades de atuação para redução do RP do gerenciamento de resíduos. O monitoramento através de indicadores de controle (IC) também permite comparaçóes entre os estabelecimentos, no que se refere à RP em cada etapa considerada, bem como do risco potencial do gerenciamento de resíduos (RPGRSS).

Com o modelo proposto, conforme a tabela 3, fica evidente a importância da avaliação minuciosa e mensuração individual dos indicadores de controle, ainda que unidades diferentes tenham obtido resultado final do nível de adequação iguais (IC1). 
Tabela 2. Resultado do RP nos EAS 1 a 10

\begin{tabular}{|l|r|r|r|r|r|r|r|r|r|r|r|}
\hline \multirow{2}{*}{$\begin{array}{l}\text { INDICADOR DE } \\
\text { CONTROLE (IC) }\end{array}$} & \multicolumn{9}{|c|}{ ESTABELECIMENTOS ASSISTENCIAIS DE SAÚDE } \\
\cline { 2 - 12 } & EAS 1 & \multicolumn{1}{|c|}{ EAS 2 } & \multicolumn{1}{|c|}{ EAS 3 } & EAS 4 & EAS 5 & \multicolumn{1}{l|}{ EAS 6 } & EAS 7 & EAS 8 & EAS 9 & EAS 10 \\
\hline ICA1 & 51,32 & 66,48 & 66,84 & 46,48 & 91,923 & 66,48 & 91,895 & 66,91 & 37,76 & 21,66 \\
\hline ICA2 & 75,08 & 50,76 & 96,132 & 75,77 & 96,804 & 75,1 & 75,24 & 75,24 & 50,76 & 21,54 \\
\hline ICB1 & 63,23 & 50,93 & 74,42 & 96,782 & 74,62 & 77,96 & 74,42 & 73,65 & 22,31 & 40,37 \\
\hline IC311 & 40,74 & 29,22 & 53,86 & 50 & 73,67 & 22,31 & 75,82 & 50 & 22,31 & 29,22 \\
\hline IC312 & 46,59 & 46,48 & 91,99 & 91,451 & 91,944 & 21,18 & 91,964 & 90,301 & 0 & 21,18 \\
\hline IC222 & 19,42 & 50 & 73,65 & 31,63 & 96,944 & 20,19 & 50 & 73,65 & 20,19 & 19,65 \\
\hline IC221 & 50 & 50 & 50 & 50 & 50 & 50 & 50 & 50 & 50 & 50 \\
\hline IC212 & 66,63 & 67,13 & 66,72 & 92,795 & 91,773 & 20,3 & 56,33 & 91,929 & 20,32 & 42,81 \\
\hline IC211 & 74,2 & 50 & 97 & 97 & 97 & 74,15 & 97 & 74,16 & 22,31 & 22,31 \\
\hline IC211A & 73,85 & 50,78 & 97 & 73,79 & 97 & 73,67 & 96,983 & 73,7 & 46,33 & 22,31 \\
\hline IC211B & 66,59 & 40,77 & 66,76 & 90,912 & 66,48 & 66,7 & 66,48 & 66,48 & & 0 & 22,12 \\
\hline IC32 & 50 & 50 & 50 & 50 & 50 & 50 & 50 & 50 & 50 & 50 \\
\hline IC31 & 46,48 & 46,8 & 74,35 & 73,65 & 74,15 & 22,31 & 74,91 & 73,65 & 22,31 & 21,75 \\
\hline IC22 & 22,31 & 47,5 & 69,56 & 33,24 & 92 & 22,31 & 47,5 & 69,56 & 22,31 & 22,31 \\
\hline IC21 & 73,53 & 72,91 & 97 & 97 & 97 & 55,35 & 96,137 & 97 & 21,5 & 47,5 \\
\hline IC3 & 47,5 & 47,5 & 70,1 & 69,56 & 69,5 & 22,31 & 70,51 & 69,56 & 22,31 & 22,31 \\
\hline IC2 & 54,52 & 53,58 & 73,65 & 74,51 & 97 & 28,01 & 73,65 & 73,65 & 20,3 & 20,3 \\
\hline IC1 & 53,18 & 50,49 & 69,3 & 69,93 & 90,981 & 25,93 & 69,93 & 69,93 & 20,3 & 20,3 \\
\hline
\end{tabular}

Fonte: elaboração própria.

\section{Tabela 3. Resultado do NAGRSS nos EAS 1 a 10}

\begin{tabular}{|l|r|r|r|r|r|r|r|r|r|r|}
\hline \multirow{2}{*}{ INDICADOR DE } & \multicolumn{10}{|c|}{ ESTABELECIMENTOS ASSISTENCIAIS DE SAÚDE } \\
\cline { 2 - 11 } CONTROLE (IC) & EAS 1 & & \multicolumn{1}{|c|}{ EAS 3 } & EAS 4 & EAS 5 & EAS 6 & EAS 7 & EAS 8 & EAS 9 & EAS 10 \\
\hline ICA1 & 48,68 & 33,52 & 33,16 & 53,52 & 8,077 & 33,52 & 8,105 & 33,09 & 62,24 & 78,34 \\
\hline ICA2 & 24,92 & 49,24 & 3,868 & 24,23 & 3,196 & 24,9 & 24,76 & 24,76 & 49,24 & 78,46 \\
\hline ICB1 & 36,77 & 49,07 & 25,58 & 3,218 & 25,38 & 22,04 & 25,58 & 26,35 & 77,69 & 59,63 \\
\hline IC311 & 59,26 & 70,78 & 46,14 & 50 & 26,33 & 77,69 & 24,18 & 50 & 77,69 & 70,78 \\
\hline IC312 & 53,41 & 53,52 & 8,01 & 8,549 & 8,056 & 78,82 & 8,036 & 9,699 & 100 & 78,82 \\
\hline IC222 & 80,58 & 50 & 26,35 & 68,37 & 3,056 & 79,81 & 50 & 26,35 & 79,81 & 80,35 \\
\hline IC221 & 50 & 50 & 50 & 50 & 50 & 50 & 50 & 50 & 50 & 50 \\
\hline IC212 & 33,37 & 32,87 & 33,28 & 7,205 & 8,227 & 79,7 & 43,67 & 8,071 & 79,68 & 57,19 \\
\hline IC211 & 25,8 & 50 & 3 & 3 & 3 & 25,85 & 3 & 25,84 & 77,69 & 77,69 \\
\hline IC211A & 26,15 & 49,22 & 3 & 26,21 & 3 & 26,33 & 3,017 & 26,3 & 53,67 & 77,69 \\
\hline IC211B & 33,41 & 59,23 & 33,24 & 9,088 & 33,52 & 33,3 & 33,52 & 33,52 & 100 & 77,88 \\
\hline IC32 & 50 & 50 & 50 & 50 & 50 & 50 & 50 & 50 & 50 & 50 \\
\hline IC31 & 53,52 & 53,2 & 25,65 & 26,35 & 25,85 & 77,69 & 25,09 & 26,35 & 77,69 & 78,25 \\
\hline IC22 & 77,69 & 52,5 & 30,44 & 66,76 & 8 & 77,69 & 52,5 & 30,44 & 77,69 & 77,69 \\
\hline IC21 & 26,47 & 27,09 & 3 & 3 & 3 & 44,65 & 3,863 & 3 & 78,5 & 52,5 \\
\hline IC3 & 52,5 & 52,5 & 29,9 & 30,44 & 30,5 & 77,69 & 29,49 & 30,44 & 77,69 & 77,69 \\
\hline IC2 & 45,48 & 46,42 & 26,35 & 25,49 & 3 & 71,99 & 26,35 & 26,35 & 79,7 & 79,7 \\
\hline IC1 & 46,82 & 49,51 & 30,7 & 30,07 & 9,019 & 74,07 & 30,07 & 30,07 & 79,7 & 79,7 \\
\hline
\end{tabular}

Fonte: elaboração própria. 
Por exemplo, para os estabelecimentos 1 e 2, cujo RPGRSS, representado pelo IC1, tem valores muito próximos (respectivamente, 53,18 e 50,49), é possível observar que possuem diferenças consideráveis em indicadores de controle distintos. Nesse sentido, o planejamento de ações prioritárias deve ser adequado a suas necessidades e portanto diferenciado para cada estabelecimento. O EAS 1 apresenta risco alto no IC222, enquanto o EAS 2 neste indicador de controle apresenta risco médio. Fica ainda mais evidente essa situação quando se compara o EAS 2 ao EAS 5, pois ambos estão na condição insatisfatória. O EAS 5 tem 90,981\% de RP, enquanto o EAS 2 apresenta um valor de 50,49\% de RP, muito próximo a transpor para a classificação satisfatória com restrição, que se atinge ao reduzir o RP para $50 \%$.

Observando a tabela 3, que apresenta a condição, nível ou grau de adequabilidade de cada IC dos EAS 1 e EAS 2, podemos concluir que o pior desempenho do EAS 1 se apresenta nos IC A2, 211, 211A e 21. E do EAS 2, nos IC A1, 212 e 21. É possível perceber, então, que há diferenças entre os indicadores de controle desses estabelecimentos no que se refere à adequação do GRSS, embora ambos se classifiquem como insatisfatórios e apresentem ao final um nível de adequação bastante próximo (respectivamente, 46,82 e 49,51).

Os EAS 9 e 10 (tabela 1 e 2) possuem resultado final idêntico do NAGRSS e no RPGRSS, apresentando diferenças significativas nos ICs, e com isso se pode propor uma estratégia específica para cada um deles (SILVA, 2010). Essas informações também são fundamentais na inspeção sanitária e direcionamento das reinspeçôes, permitindo aumentar o controle dos riscos sanitários envolvidos nas operações fiscalizadas.

O EAS 4 foi avaliado com valor de 53,52 no ICA1, que representa segregação e identificação dos resíduos; obteve avaliação no valor de 7,205 no IC212, que representa o transporte externo e destino final. Comparativamente, embora com mesmo resultado final $(30,77)$, o EAS 7 obteve avaliações análogas nos mesmos indicadores de controle, ou seja, valor 8.01 no ICA1 e valor 43,63 no IC212.

Ressalta-se a importância da individualização dos indicadores de controle, porque permite trabalhar a deficiência particular em cada um dos ICs. Por exemplo: é pouco interessante segregar e identificar corretamente todos os resíduos, se no momento do transporte e destino final este trabalho é perdido, conduzindo ao mesmo resultado que transportar e destinar, em melhores condições, resíduos não segregados e identificados incorretamente. 
Considerando-se as questôes postas e os resultados do modelo sistêmico para inspeções de RSS, o Fuzzy Visa se apresenta coerente com a realidade. A adoção de indicadores permite a construção de um instrumentode apoio à decisão, capaz de comparar os serviços e o monitoramento dos mesmos, bem como auxiliar na simulação de metodologias de gestão de resíduos.

Embora o modelo possa significar um avanço na atuação da vigilância sanitária, deve ser considerado que foram utilizados poucos dados e mais testes devem ser realizados.

\section{Conclusões}

O propósito fundamental deste trabalho foi apresentar um sistema baseado em lógica fuzzy aplicado à inspeção sanitária A partir do conceito de risco potencial, desenvolveu-se um sistema capaz de avaliar os fatores críticos para a vigilância sanitária, no planejamento de suas ações e na sua capacidade de atuar sobre os riscos à saúde e de intervir nos problemas sanitários.

O instrumento classifica o grau de risco do estabelecimento gerador de RSS e a condição de satisfação em função do seu gerenciamento. Além disso, se abrem novas perspectivas, como a criação de um cenário de risco regional de importância para a saúde pública. Este pode indicar ações a serem realizadas não apenas no âmbito da vigilância sanitária, mas em outros setores, principalmente porque a questão dos resíduos de serviços de saúde extrapola a área da saúde pública, dada sua transversalidade, impactando, por exemplo, o meio ambiente.

Os testes de validação do modelo fuzzy proposto foram realizados buscando simular a inspeção em dez EAS do estado do Rio de Janeiro, sendo utilizados dados expressos em relatórios de inspeções já realizadas. A aplicação dos testes e os resultados encontrados no funcionamento do modelo fuzzy indicam que o mesmo tornaria possível a sistematização e racionalização das avaliações de risco, contemplando os aspectos ambíguos e incertos inerentes às avaliações da inspeção sanitária de RSS, como satisfatório, insatisfatório, alto, baixo e outros, mostrando-se um modelo aplicável nas inspeçōes sanitárias.

A adoção do modelo pode ainda melhor aproveitar os recursos humanos capacitados em inspeção de RSS, aumentando não só a qualidade da inspeção, quanto a quantidade de estabelecimentos inspecionados, o que é importante em vista do grande número de serviços existentes no estado. A ausência de 
inspeção, ou intervalos muito grandes, pode ser considerada um fator de risco para o gerenciamento dos RSS, com consequências sanitárias, sobretudo porque a presença da fiscalização reduz os perigos de descumprimento dos preceitos legais e resulta na melhoria da qualidade do gerenciamento. Pela simplicidade de utilização, pode ainda ser utilizado como um sistema de autoinspeção, inclusive hospedado via web, que proporcionaria ao estabelecimento se autoavaliar, passando a planejar a correção de suas inconformidades, antecipando-se às inspeções oficiais.

O modelo também busca racionalizar o pensamento humano, presente em todas as inspeções sanitárias. Espera-se obter um comportamento padronizado quanto aos resultados, imprimindo um tratamento equivalente das informações e ampliando a qualificação do processo de fiscalização. Em consequência, o modelo poderá contribuir na manutenção de uma saúde pública de qualidade, com redução do impacto ambiental dos RSS no ambiente, mas especificamente no campo da vigilância sanitária. A construção de um instrumento de avaliação de RP poderá ser decisiva no desenho da realidade e do gerenciamento dos RSS no estado e na avaliação do grau de atendimento às normas sanitárias.

O modelo representa o raciocínio do agente fiscalizador e sua aplicação resultará na exposição do estágio dos EAS no estado do Rio de Janeiro. Ele significa também a complexidade de confluência do atendimento às normas e regulamentos frente ao cenário atual. Embora tenha buscado adotar critérios objetivos, é sempre possível haver entendimentos diferenciados, uma vez que considerou-se a experiência da equipe de inspeção de RSS da vigilância sanitária do estado.

A construção e o uso de um modelo com base científica, no processo de avaliação de risco da inspeção de RSS, é uma busca necessária que através deste estudo divulga a metodologia de análise e o conhecimento utilizado, acreditando ser possível a melhoria da qualidade do gerenciamento dos resíduos, através da interação com o processo de avaliação e ações positivas, levando a um cenário de integração entre o estabelecimento fiscalizado e o agente fiscalizador.

Sobre o modelo construído, recomenda-se o desenvolvimento da interface computacional que motive sua aplicação direta nas inspeções e de um sistema de informação que facilite o monitoramento dos serviços. A criação de cenários situacionais e regionais minimizaria a necessidade de conhecimentos específicos sobre fuzzy ou sobre o software MATLAB/SIMULINK. 
Espera-se que este trabalho sirva de estímulo e referencial ao desenvolvimento de instrumentos que possam contribuir e apoiar a atuação da Vigilância Sanitária, buscando ampliar conhecimentos e produzir formas, cada vez mais efetivas, de gerenciar riscos. ${ }^{3}$

\section{Referências}

BRASIL.Ministério da Saúde. Resolução RDC/ANVISA nº. 306, de 07 de dezembro de 2004. Dspõe sobre o regulamento técnico para o gerenciamento dos resíduos de serviços de saúde. Diário Oficial da União, Brasília, DF, 10 dez. 2004. Seção 1, p. 49.

COSTA, E. A. Vigilância sanitária: proteção e defesa da saúde. 1. Ed. São Paulo: Hucitec: Sobravime, 1998.

COSTA, E. A. Vigilância sanitária: proteção e defesa da saúde: São Paulo: HucitecSobravime, 2004.

LEITE, H. J. D. Vigilância sanitária em serviços de saúde: risco e proteção da saúde em serviços de hemodiálise. 2007. Tese (Doutorado) - Instituto de Saúde Coletiva, Universidade Federal da Bahia, Salvador, 2007.

ZADEH, L. A. fuzzy sets. Information and Control. New York, v. 8, n. 3, p. 338-353, 1965. COSTA, E. A. Vigilância sanitária: proteção e defesa da saúde. In: ROUQUARIOL, M. Z.; ALMEIDA FILHO, N. (Org.). Epidemiologia e saúde. Rio de Janeiro: Medsi, 2003.

AMORIM, L. A. et al. Agente de Suporte a Decisão Multicritério com Soma Ponderadafuzzy em Gestão Pública Participativa: Um Estudo de Caso em Gestão Ambiental. Revista Brasileira de Sistemas de Informação, Vol 8 No 3 pp 28-41, Rio de Janeiro, editada em 2015 pela Sociedade Brasileira de Computação.

JANG, J.-S. R.; SUN, C.-T.; MIZUNAMI, E.; Neuro-fuzzy ans Soft Computing, 1997 Prentice Hall.

LIMA, F. R.; CARPINETTI, L. C. R.; Uma comporação entre os métodos Topsis e FuzzyTopsis no apoio à tomada de decisão multicritério para seleção de fornecedores. Revista Gestão \& Produção, v. 22, n. 1 p. 17-34. São Carlos, 2015.

JENSEN F.V.; NIELSEN T.N. Bayesian networks and decision graphs. $2^{\text {nd }}$ ed. New York: Springer, 2007.

SILVA, N.F.C.; Fuzzy Visa: modelo de avaliação de risco da vigilância sanitária para inspeção de resíduos de serviços de súde utilizando lógica fuzzy. (Tese) Doutorado em Saúde Coletiva, Instituto d Medicina Social Universidade do Estado do Rio de Janeiro, 2010. 
${ }^{1}$ Uma discussão sobre lógica fuzzy e suas aplicaçôes na área de saúde pode ser vista em Amorim et al. (2015), Lima e Carpinetti (2015) e Jang, Sun e Mizunami (1997).

${ }^{2}$ A situação do EAS 5 está sujeita a interdição, tamanho e seu risco potencial.

${ }^{3}$ N.F.C. da Silva foi responsável pela construção do artigo e desenvolvimento da metodologia.

C.M.M. Vianna foi responsável pela revisão da avaliação da tecnologia proposta na área da saúde. F.S.G. de Oliveira realizou a revisão da metodologia fuzzy. G.B.G. Mosegui realizou a revisão das metodologias de vigilância sanitária. M.P.S. Rodrigues realizou a revisão do modelo computacional. 
Fuzzy Visa: a fuzzy logic model for risk assessment of Health Surveillance for health services waste inspection

The sanitary inspection of Health Services Waste Management (HSWM) lacks a standardized work methodology that provides a secure basis for evaluating potential risk (PR). This study aimed to propose an instrument based on fuzzy logic that could standardize inspection and generate indicators of sanitary control. The model was developed with 18 subsystems related to HSWM obtained from the assembly of elements identified in the inspection as those that interfere with the assessment and permeate all stages of HSWM. The validation of the model was conducted in 10 health establishments in Rio de Janeiro between May to November 2009. The results obtained by this model were consistent with the diagnoses made by experts and clearly indicated the possibility of the systematization and rationalization of PR assessments. This decision support system makes management and planning of actions more efficient in PR evaluation.

> Keywords: health services waste; fuzzy logic; health surveillance; sanitary inspection; decision support system. 